\title{
Application of Evolutionary Algorithms in Guaranteed Parameter Estimation
}

\author{
Thilo Goerke, Sebastian Engell \\ Process Operations and Dynamics Group \\ Technische Universität Dortmund \\ Dortmund, Germany \\ thilo.goerke@bci.tu-dortmund.de, sebastian.engell@,bci.tu-dortmund.de
}

\begin{abstract}
Model-based optimization and control is becoming more and more important in the process industries and in general. Modelling almost always involves the estimation of parameters from available data. The parameter estimation problem is usually posed as the minimization of the prediction error or the maximization of the likelihood function. If the uncertainty of the measurements taken from a real process is assumed to be an interval around the measured values, a set of parameter vectors exists that is able to describe the behavior of the systems within these uncertainties. Guaranteed parameter estimation deals with the problem of determining all parameter vectors that are compatible with uncertain observations. The solution of guaranteed parameter estimation problems for nonlinear dynamic models is computationally very demanding. In this contribution we present a memetic algorithm that determines the sets of feasible model parameters efficiently. It is applied to the estimation of kinetic parameters of a model that describes a copolymerization reaction. In the memetic algorithm, the fitness evaluation is based on the distance of the feasible solutions to each other, thus the presented approach is not restricted to a specific type of models.
\end{abstract}

Keywords-guaranteed parameter estimation, memetic algorithm, measurement uncertainty

\section{INTRODUCTION}

When developing dynamic models of plants in the process industries, two main steps have to be executed. The first step is to decide about the model structure, i.e. should a black box model, a rigorous model or any other type be chosen to describe the mechanisms of interest in the process. Also the complexity of the model or the phenomena that are taken into account have to be chosen. The second step is to parameterize the selected model structure. The choice of the model structure is made in an iterative process where models are formulated, parameters of the model are chosen or fitted to data, and the models are validated against observations. Usually not all model parameters are available from the literature or from independent experiments, so fitting a model to data by adapting model parameters is needed. One issue in dealing with experimental data is the uncertainty of the data which can be caused by several reasons, e.g. inaccuracy of the measurement device and noise (stochastic errors) in the measurements. Secondly structural plant-model mismatch has to be taken into account which is caused by effects that are not reflected in the model or wrong assumptions on the basic laws that are chosen. Usually, one set of parameters is estimated, using a least squares or maximum likelihood criterion and the uncertainties are reflected in confidence intervals of the parameters. These confidence intervals are hyper ellipsoids in the parameter space that are computed based on sensitivities of the experiments to the parameters, so they are valid for small errors around a nominal model. A more rigorous way of computing the uncertainties in model parameters is guaranteed parameter estimation [1]. The idea is to determine the complete sets of model parameters which are compatible with the observations within the known uncertainties of the measurements. To determine these sets is computationally very demanding, as the problem is nonlinear and nonconvex. A lot of current research discusses how to compute approximations of the true sets of the possible parameters efficiently, e.g. [2] and [3]. In this contribution we present a new approach to determining the feasible parameter sets of given models for uncertain measurement data. The approach employs an evolutionary algorithm in combination with a local gradient-based optimization (memetic algorithm). The method is demonstrated for the example of a kinetic model of a complex polymerization process. The paper is organized as follows. In Section 2 the guaranteed parameter estimation problem is introduced, Section 3 deals with the memetic algorithm and introduces the optimization criterion. Section 4 explains the modelling of the copolymerization example and the measurements. In section 5, the results of the guaranteed parameter estimation are presented, in Section 6 conclusions are drawn and an outlook on future work is given.

\section{THE GUARANTEED PARAMETER ESTIMATION PROBLEM} form

In this contribution we assume that a dynamic model in the

$$
y(t)=f\left(u(\tau), 0 \leq \tau \leq t, x_{0}, p_{f}, p_{e}\right)
$$

has been set up where y denotes the output vector of the model, $\mathrm{u}$ describes the input vector, $\mathrm{x}_{0}$ describes the initial state, $\mathrm{p}_{\mathrm{f}}$ represents the vector of fixed parameters, $p_{e}$ contains the parameters that have to be estimated and $t$ denotes time. Usually the solution of a classical parameter estimation problem is a unique parameter set $p_{e}^{*}$ that is calculated by the following optimization problem

$$
\begin{aligned}
& p_{e}^{*}=\min _{p_{e}} \sum_{i=1}^{N}\left(y_{m}\left(t_{i}\right)-y\left(t_{i}\right)\right)^{2} \\
& \text { s.t. } \mathrm{y}(\mathrm{t})=\mathrm{f}\left(\mathrm{u}(\tau), 0 \leq \tau \leq \mathrm{t}, \mathrm{x}_{0}, \mathrm{p}_{\mathrm{f}}, \mathrm{p}_{\mathrm{e}}\right)
\end{aligned}
$$




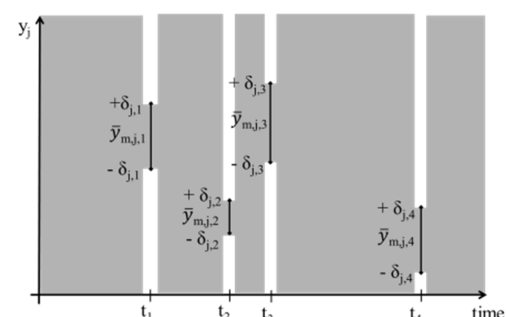

Figure 1: Possible model predictions including uncertainty in four measurements

which depends on the difference of the model prediction $y\left(t_{i}\right)$ and the measurement $\mathrm{y}_{\mathrm{m}}\left(\mathrm{t}_{\mathrm{i}}\right)$ at several points in time. As the measurements contain measurement errors and hence are uncertain, one has to consider the effect of the uncertainty on the estimation problem. Figure 1 illustrates the uncertainty of the measurements.

Each measurement of the $\mathrm{j}$-th measured variable at the $\mathrm{i}$-th point in time is assumed to be a tuple consisting of the mean values $\bar{y}_{m, j, i}$ and stochastic errors within the intervals $\left[-\delta_{\mathrm{j}, \mathrm{i}}, \delta_{\mathrm{j}, \mathrm{i}}\right]$. Fig. 1 shows the space of possible predictions of the models that are restricted at the points in time where the measurements have been taken and even at these points in time there are intervals of possible values through which the trajectories must pass. The extension of the measurement values to intervals leads to a modification of the optimization problem, as not a unique solution can to be found but every set of parameters that makes the trajectories pass through the measurement intervals is a solution. We define the set of parameters, for which the simulated trajectories pass through the measurement intervals, as $\mathrm{P}^{*}$ which is subject to the following conditions

$$
\begin{array}{r}
\mathrm{P}^{*}=\left\{\mathrm{p}_{e} \in\left[\mathrm{p}_{l}, \mathrm{p}_{u}\right] \mid \mathrm{f}\left(\mathrm{u}(\tau) .0 \leq \tau \leq \mathrm{t}, x_{0}, p_{f}, p_{e}\right) \in\right. \\
\left.\left[y_{m, j}\left(\mathrm{t}_{i}\right) \pm \delta_{j, i}\right]\right\}(3)
\end{array}
$$

$\mathrm{p}_{1}$ and $\mathrm{p}_{\mathrm{u}}$ denote lower and upper values for each estimated parameter that may be given from prior knowledge (e.g. certain parameters cannot be negative). In the following sections we define a feasible solution as a parameter set that is an element of $\mathrm{P}^{*}$. In order to determine $\mathrm{P}^{*}$ in the parameter space we propose a memetic algorithm which is presented in the following section.

\section{THE MEMETIC ALGORITHM}

The memetic algorithm used in this work is based on the evolutionary algorithm from [4] and based on the memetic algorithm proposed in [5] where the combination of an evolutionary algorithm with a conventional local optimization solver was proposed and applied to chemical engineering design problems. The underlying ideas are to use the evolutionary part of the algorithm to explore the parameter space, while the local optimization deals with the search in a specific region of the search space as it is more efficient in finding local minima with respect to real variables than an evolutionary algorithm. This strategy is efficient for highly non-convex optimization problems that potentially may have disconnected solution sets with local optima. The algorithm is also able to solve problems to compute feasible regions under constraints as e.g. finding product ranges for specific polymerization processes as presented in [6]. The search space in the case of the guaranteed parameter estimation is defined by a k-dimensional cube, which is bounded by $p_{k, 1}$ and $p_{k, u}$ of the $\mathrm{k}$ parameters that are

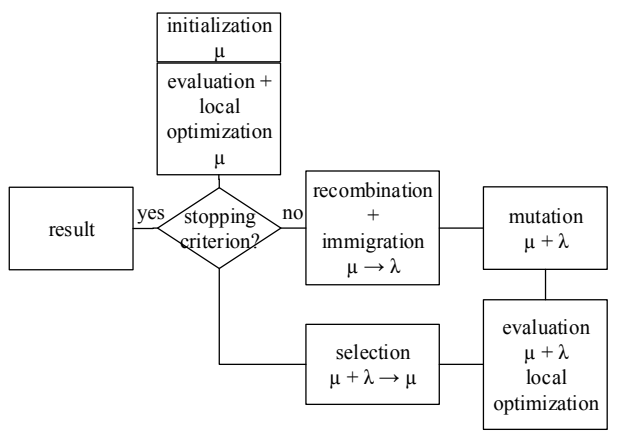

Figure 2: Scheme of the memetic algorithm [6]

determined from the measurements. In this contribution we applied a $[\mu+\lambda, \kappa]$ - algorithm following the notation in [7]. An immigration of individuals during the recombination step of the algorithm is added to enable a better coverage of the search space also for higher numbers of generations. The structure of the memetic algorithm is depicted in Fig. 2.

The first generation consists of $\mu$ individuals that are drawn assuming a uniform distribution within the search space. The evaluation after the initialization includes also a local optimization step and is used to determine if an individual is part of $\mathrm{P}^{*}$, to calculate the maximum distance from the feasibility boundaries and to maximize the volume of the feasible parameter space. The first two objectives are evaluated by the distance $d_{b}$ of an individual characterized by the parameter set $p_{e}$ towards the feasibility boundary. The distance function is defined as

$$
\begin{gathered}
d_{b}\left(p_{e}\right)=\max \left(\begin{array}{c}
0, p_{l, k}-p_{e, k}, p_{e, k}-p_{u, k}, \\
\left(y_{m, j}\left(\mathrm{t}_{i}\right)-\delta_{j, i}\right)-\mathrm{f}\left(\mathrm{u}, \mathrm{u}_{0}, \mathrm{p}_{f}, \mathrm{p}_{e}, \mathrm{t}_{i}\right), \\
\mathrm{f}\left(\mathrm{u}, \mathrm{u}_{0}, \mathrm{p}_{f}, \mathrm{p}_{e}, \mathrm{t}_{i}\right)-\left(y_{m, j}\left(\mathrm{t}_{i}\right)+\delta_{j, i}\right)
\end{array}\right) \\
\operatorname{for}\left\{\begin{array}{c}
\forall i \in\left[1, \ldots, N_{\text {measurement }}\right] \\
\forall j \in\left[\begin{array}{c}
1, \ldots, \text { types of measurements }] \\
\forall \mathrm{k} \in\left[1, \ldots, \mathrm{N}_{\text {parameters }}\right]
\end{array}\right.
\end{array}\right.
\end{gathered}
$$

The distance towards the boundary equals 0 if the constraints defined by the upper and lower parameter limits or by the measurements are fulfilled, otherwise the value is positive and represents the maximum distance towards the feasibility boundary. In order to maximize the volume of the feasible parameter space a second evaluation function is introduced, which calculates the distance of the the feasible solutions towards all other feasible solutions found during the execution of the memetic algorithm. The second evaluation criterion is

$$
d_{\text {ind }, P *}\left(p_{e}\right)=\sum_{a=1}^{N_{\text {feas }}} \sum_{k=1}^{N_{\text {parameters }}} \frac{\left|p_{k, a}-p_{k, e}\right|}{N_{\text {feas }}} .
$$

This function is used by the local optimization solver as the cost function in order to increase the volume of $\mathrm{P} *$. By a different local optimization, the number of feasible individuals within the search space is increased. Depending on the feasibility of the individual the local search algorithm and the 
cost function differ. If the individual is infeasible $\left(d_{b}\left(p_{e}\right)>0\right)$, (4) is used as the cost function value in a minimization by a call of the KNITRO solver taken from the TOMLAB toolbox Ver. 7.7. Otherwise the CONOPT solver, taken also from the TOMLAB suite, is used to maximize (5) under the condition that (4) is non-positive, which is used as a constraint. The reason for choosing two different solvers is the different performance of both solvers for the different optimization problems. The presented strategy of the local optimization is also applied during the repeated evaluation in the algorithm.

During the recombination, $90 \%$ of the $\lambda-\mu$ individuals are generated by exchanging the information of two arbitrarily chosen individuals. The remaining $10 \%$ are generated by the initialization mechanism, which can be interpreted as an immigration of individuals to the current population. The following mutation is realized by applying a step size based method presented in [8]. As shown in (6)-(8) the mutation step size is calculated based on two numbers taken from a normal distribution with a mean value of 0 and a standard deviation of 1. The first one, $\mathrm{N}_{\mathrm{gen}}$, is calculated globally for each generation, while the second one, $\mathrm{N}_{\mathrm{k}, \mathrm{n}}$, differs for each of the $\mathrm{n}$ individuals in a generation and for each entry of the corresponding individual.

$$
\begin{aligned}
& \Delta_{\text {mut }, \text { new }_{k, n}}=N_{k, \mathrm{n}} \cdot \Delta_{\text {mut }, \text { old }} \cdot e^{\tau_{\text {gen }} N_{\text {gen }}+\tau_{\text {ind }} N_{k, n}} \\
& \tau_{\text {gen }}=\left(2 N_{\text {parameters }}\right)^{-0.5} \\
& \tau_{\text {ind }}=\left(2\left(N_{\text {parameters }}\right)^{0.5}\right)^{-0.5}
\end{aligned}
$$

This mechanism results in a step size which is decreasing with the number of generations, which narrows the search for higher numbers of generations. The step size of the mutation is restricted to the interval $\left[\Delta_{m u t, l, k}, \Delta_{m u t, u, k}\right]$ in order to avoid a negligible mutation or a competition with the recombination mechanism, which is responsible for large steps in the search space. The direction of the mutation is oriented towards better areas of the search space by the selection step. If the upper and lower limits of the parameters are exceeded due to the recombination or the mutation, a mirroring at the corresponding boundary is performed to avoid the creation of a priori infeasible individuals. After the mutation step, an evaluation, including the local optimization strategy, of the existing $\mu+\lambda$ individuals is performed.

The following selection step is performed based on a two stage tournament selection. In order to adjust the selection pressure, subgroups with a size of $\mathrm{n}_{\text {sub }}$ individuals taken from the current generation are generated. The scheme is shown in Fig. 3. Applying this scheme, either the best infeasible individual or the best feasible individual is selected to form the following parent generation. During the selection individuals with a higher lifetime than $\kappa$ are discarded. In order to avoid the loss of the information of feasible solutions, every feasible individual is stored in an archive, which is used in the calculation of (5). After the selection step, the current lifetime of each selected individual is increased by one. The stopping criterion used in the proposed algorithm is the number of generations investigated. The total number of individuals in the initial generation depends on the number of parameters to be estimated in order to achieve a reliable coverage of each axis in the k-dimensional search space. The reproduction rate was taken from [7]. The choice of the maximum lifetime is a tradeoff between the mortality rate of the individuals and the storage capacity of good individuals over a certain amount of generations in order to avoid both a search within the zone of attraction of a single local optimum and the unproductive investigation of specific areas within the search space. The parameters chosen for the investigation of the case study are shown in Tab. 1. During the execution of the algorithm the degrees of freedom and the measurements are scaled between 0 and 1 according to equation (9) in order to avoid numerical issues and to simplify the representation of the results. The minimum and maximum values of the degrees of freedom are taken from the assumed boundaries.

$$
x_{\text {scaled }}=\frac{x_{\text {unscaled }}-x_{\min }}{x_{\max }-x_{\min }}
$$

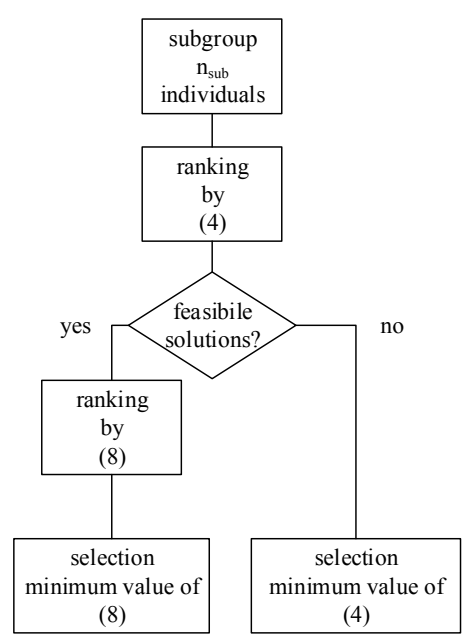

Figure 3: Scheme of the two-stage selection step [6]

Table 1: Parameters used in the memetic algorithm [6]

\begin{tabular}{|c|c|c|}
\hline parameter & description & value \\
\hline$\mu$ & $\begin{array}{c}\text { number of individuals in the } \\
\text { parental generation }\end{array}$ & $\begin{array}{c}100 \\
\mathrm{~N}_{\text {parameters }}\end{array}$ \\
\hline$\frac{\lambda}{\mu}$ & reproduction rate & 7 \\
\hline$\kappa$ & $\begin{array}{c}\text { maximum lifetime of an } \\
\text { individual }\end{array}$ & 5 \\
\hline $\mathrm{n}_{\text {init }}$ & $\begin{array}{c}\text { newly initialized individuals } \\
\text { during recombination }\end{array}$ & $\frac{\lambda-\mu}{10}$ \\
\hline$\Delta_{m u t, l, k}$ & $\begin{array}{c}\text { lower step size limit during } \\
\text { mutation }\end{array}$ & $\frac{u_{k, u}}{100}$ \\
\hline$\Delta_{m u t, u, k}$ & $\begin{array}{c}\text { upper step size limit during } \\
\text { mutation }\end{array}$ & $\frac{u_{k, u}}{10}$ \\
\hline $\mathrm{n}_{\text {sub }}$ & $\begin{array}{c}\text { size of subgroup during } \\
\text { selection }\end{array}$ & $\begin{array}{c}\frac{\mu}{5} \\
\text { individuals }\end{array}$ \\
\hline $\mathrm{n}_{\text {gen }}$ & $\begin{array}{c}\text { maximum number of } \\
\text { evaluated generations }\end{array}$ & 30 \\
\hline
\end{tabular}

\section{COPOLYMERIZATION CASE STUDY}

In order to test the novel guaranteed parameter estimation technique a copolymerization process was chosen where six kinetic parameters must be estimated. The investigated process 
Table 2: Reaction scheme of a radical polymerization

\begin{tabular}{|c|c|}
\hline reaction step & reaction equation \\
\hline initiator decomposition & $\mathrm{I} \stackrel{\mathrm{k}_{\mathrm{i}}(\mathrm{T})}{\longrightarrow} 2 \mathrm{R}_{0} *$ \\
\hline chain initiation & $\begin{array}{l}\mathrm{R}_{0} *+\mathrm{M}_{1} \stackrel{\mathrm{k}_{\mathrm{i}, 1}(\mathrm{~T})}{\longrightarrow} \mathrm{P}_{1, \mathrm{M} 1} * \\
\mathrm{R}_{0} *+\mathrm{M}_{2} \stackrel{\mathrm{k}_{\mathrm{i}, 2}(\mathrm{~T})}{\longrightarrow} \mathrm{P}_{1, \mathrm{M} 2} *\end{array}$ \\
\hline propagation & $\begin{array}{l}\mathrm{P}_{\mathrm{n}, \mathrm{M} 1} *+\mathrm{M}_{1} \stackrel{\mathrm{k}_{\mathrm{p} 11}(\mathrm{~T})}{\longrightarrow} \mathrm{P}_{\mathrm{n}+1, \mathrm{M} 1} * \\
\mathrm{P}_{\mathrm{n}, \mathrm{M} 1} *+\mathrm{M}_{2} \stackrel{\mathrm{k}_{\mathrm{p} 12}(\mathrm{~T})}{\longrightarrow} \mathrm{P}_{\mathrm{n}+1, \mathrm{M} 2} * \\
\mathrm{P}_{\mathrm{n}, \mathrm{M} 2} *+\mathrm{M}_{1} \stackrel{\mathrm{k}_{\mathrm{p} 21}(\mathrm{~T})}{\longrightarrow} \mathrm{P}_{\mathrm{n}+1, \mathrm{M} 1} * \\
\mathrm{P}_{\mathrm{n}, \mathrm{M} 2} *+\mathrm{M}_{2} \stackrel{\mathrm{k}_{\mathrm{p} 22(\mathrm{~T})}}{\longrightarrow} \mathrm{P}_{\mathrm{n}+1, \mathrm{M} 2} *\end{array}$ \\
\hline $\begin{array}{l}\text { termination by } \\
\text { combination }\end{array}$ & $\begin{array}{c}\mathrm{P}_{\mathrm{n}, \mathrm{M} 1} *+\mathrm{P}_{\mathrm{m}, \mathrm{M} 1} * \stackrel{\stackrel{\mathrm{k}_{\mathrm{tcl} 1}(\mathrm{~T})}{\longrightarrow} \mathrm{D}_{\mathrm{n}+\mathrm{m}}}{\longrightarrow} \mathrm{k}_{\mathrm{n}, \mathrm{M} 1 \text { or } \mathrm{M} 2} *+\mathrm{P}_{\mathrm{m}, \mathrm{M} 2 \text { or } \mathrm{M} 1} * \stackrel{\mathrm{k}_{\mathrm{tc} 1}(\mathrm{~T})}{\longrightarrow} \mathrm{D}_{\mathrm{n}+\mathrm{m}} \\
\mathrm{P}_{\mathrm{n}, \mathrm{M} 2} *+\mathrm{P}_{\mathrm{m}, \mathrm{M} 2} * \stackrel{\text { ktc2 }(\mathrm{T})}{\longrightarrow} \mathrm{D}_{\mathrm{n}+\mathrm{m}}\end{array}$ \\
\hline $\begin{array}{c}\text { termination by } \\
\text { disproportionation }\end{array}$ & $\begin{array}{c}\mathrm{P}_{\mathrm{n}, \mathrm{M} 1} *+\mathrm{P}_{\mathrm{m}, \mathrm{M} 1} * \stackrel{\mathrm{k}_{\mathrm{td} 1}(\mathrm{~T})}{\longrightarrow} \mathrm{D}_{\mathrm{n}}+\mathrm{D}_{\mathrm{m}} \\
\mathrm{P}_{\mathrm{n}, \mathrm{M} 1 \text { or } \mathrm{M} 2} *+\mathrm{P}_{\mathrm{m}, \mathrm{M} 2 \text { or } \mathrm{M} 1} * \stackrel{\mathrm{k}_{\mathrm{td} 12}(\mathrm{~T})}{\longrightarrow} \mathrm{D}_{\mathrm{n}}+\mathrm{D}_{\mathrm{m}} \\
\mathrm{P}_{\mathrm{n}, \mathrm{M} 2} *+\mathrm{P}_{\mathrm{m}, \mathrm{M} 2} * \stackrel{\mathrm{k}_{\mathrm{td} 2}(\mathrm{~T})}{\longrightarrow} \mathrm{D}_{\mathrm{n}}+\mathrm{D}_{\mathrm{m}}\end{array}$ \\
\hline
\end{tabular}

is a polymerization in a stirred tank where a reaction system that consists of two different monomers that react following a radical polymerization mechanism in the presence of an initiator. The model we chose to describe the reaction mechanism is based on the so-called terminal model that is commonly used to model radical polymerizations, see e.g. [9]. The reaction scheme is shown in Tab. 2. The initiator I decomposes into two radicals $\mathrm{R}_{0}$, which act as the starting point for the generation of active chains $\mathrm{P}_{\mathrm{i}, \mathrm{MX}}$. The growth of the chains happens by the propagation steps where one monomer unit is added to the chain. Active chains become inactive ones, labeled $\mathrm{D}$, when they lose their radical. The deactivation can be caused by different mechanisms by which two active chains are deactivated. In total 13 reactions are taking place in the process, resulting in 26 kinetic parameters, where an Arrhenius approach is used to describe the temperature dependency of the reaction rates. Previous investigations, e.g. in [6], showed that the number of reaction rates that are needed to describe a copolymerization system can be reduced to six. These six reaction rates are two reactivity ratios $r_{1}$ and $r_{2}$, that describe the ratios of homopolymerization and crosspropagation, the homopropagation rates $\mathrm{k}_{\mathrm{p} 11}, \mathrm{k}_{\mathrm{p} 22}$ and two general termination rates $\mathrm{k}_{\mathrm{t} 1}$ and $\mathrm{k}_{\mathrm{t} 2}$. Due to the statistical nature of the reaction mechanism, the resulting product cannot be described by single product quality. One of the accepted indicators for the product quality of a polymer product is the chain length distribution. Two indicators that describe the distribution are the corresponding number average (NA) and the weight average (WA). In order to reduce the computational overhead for the calculation of these indicators, the method of moments is applied. The kinetic model consists in total of 23 ODEs describing the concentrations of the small molecules and the moments up to the $2^{\text {nd }}$ moment of the active and inactive chains. The set of ODEs can be found in [9].

The process considered in this contribution takes place in a stirred tank reactor that is operated in an isothermal mode of operation, which results in only six kinetic parameters that have to be estimated. We assume to have the data of three different (simulated) experiments available. The three experiments are described by different feeding strategies of the monomers. For all experiments a prefilling of the reactor with all components, a solvent, initiator and, depending on the chosen experiment, one or two monomers takes place. The data consists of one copolymerization experiment (feeding of two monomers) and one homopolymerization experiment for each monomer. The mean values of the measurements of the experiments were generated by using the same model as was used for the parameter estimation, which results from our assumption that the model structure represents the reality correctly. The measurement devices are assumed to be a discontinuously operated concentration measurement device for both monomers and a discontinuously operated device to determine the number average and weight average of the resulting chain length distribution. The uncertainty of the concentration measurement device is assumed to be $10 \%$ of the mean value, the uncertainty in the device measuring the chain length distribution is assumed to be $5 \%$ of the measured value. While the latter device is assumed to be available only in the homopolymerization experiments, the first one is available in all experiments. The reason for the limitation is to investigate the influence of available data on the quality of the guaranteed parameter estimation and to show the potential of the guaranteed parameter estimation to determine which information is required to arrive at a reasonably accurate model. Therefor we apply two different runs of the algorithm. The first one is performed using the information taken only from the copolymerization experiment, while in the second run the data of all three experiments is used. This will highlight the potential of guaranteed parameter estimation for the decision whether a model with the determined parameters is sufficiently accurate or whether further data is needed. The generated data for the guaranteed parameter estimation is shown in Figs. 4 to 6. The concentration measurements for both monomers are taking place at the same time. During the copolymerization experiment the concentration measurement of monomer 1 is assumed to take place every 10 minutes for the first $200 \mathrm{~min}$, while the measurement of the concentration of the second monomer is spread over the whole duration of the experiment. The duration of every experiment is fixed to $2000 \mathrm{~min}$. The gap between each concentration measurement in the homopolymerization experiments is set to 50 minutes, similar to the time gap for the measurement of the chain length distribution. The amounts of prefilled components are assumed to be known. Thus the starting points are not shown in the concentration profiles (Figs. 4-6). The trajectories of the experiments are obtained by applying the nominal values shown in Tab. 3, which also contains the upper and lower limits used for scaling the parameters according to (9).
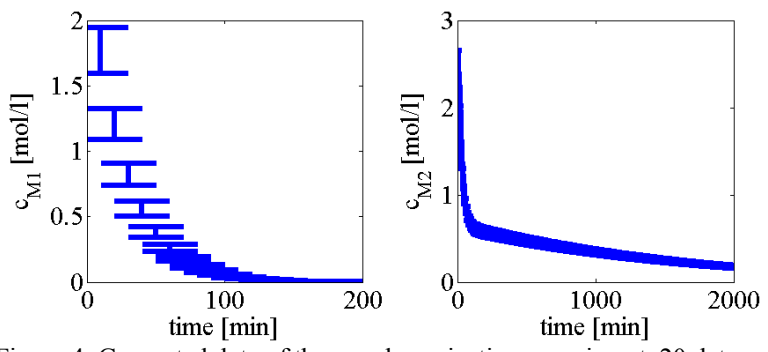

Figure 4: Generated data of the copolymerization experiment; 20 data points for $\mathrm{c}_{\mathrm{M} 1}$ and 200 data points for $\mathrm{c}_{\mathrm{M} 2}$ 

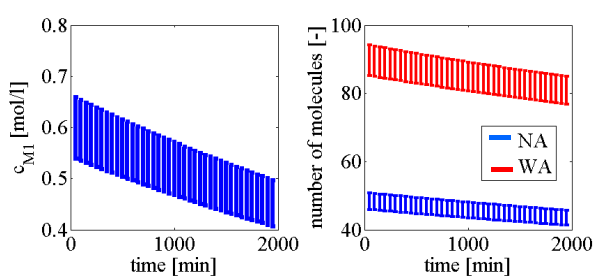

Figure 5: Generated data of the homopolymerization experiment of M1; 40 data points from the concentration measurement and from the chain length distribution
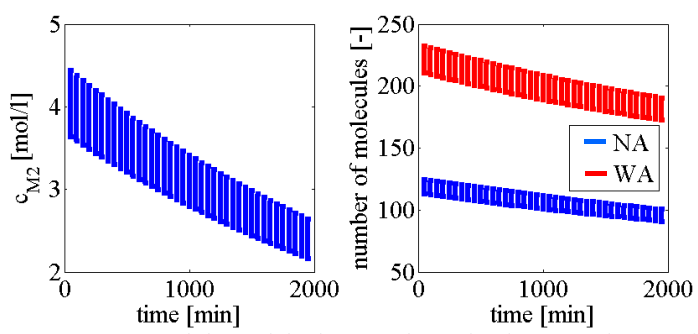

Figure 6: Generated data of the homopolymerization experiment of M2; 40 data points from the concentration measurement and from the chain length distribution

Table 3: Parameters limits and values used for generating the experimental data and the scaling according to (9) in Figs. 7-11

\begin{tabular}{|c|c|c|c|}
\hline parameter & lower limit & nominal value & upper limit \\
\hline $\mathrm{r}_{1}$ & $10^{-4}$ & $10^{-4}$ & $10^{4}$ \\
\hline $\mathrm{r}_{2}$ & $10^{-4}$ & $10^{-2}$ & $10^{4}$ \\
\hline $\mathrm{k}_{\mathrm{p} 11}$ & $10^{4}$ & $10^{4.23}$ & $10^{8}$ \\
\hline $\mathrm{k}_{\mathrm{p} 22}$ & $10^{4}$ & $10^{4.31}$ & $10^{8}$ \\
\hline $\mathrm{k}_{\mathrm{t} 1}$ & $10^{8}$ & $10^{12}$ & $10^{15}$ \\
\hline $\mathrm{k}_{\mathrm{t} 2}$ & $10^{8}$ & $10^{12.30}$ & $10^{15}$ \\
\hline
\end{tabular}

\section{RESULTS OF THE GUARANTEED PARAMETER ESTIMATION}

The information shown in Fig. 4 is used in the first run of the algorithm. As information about the chain length distribution is missing, the experiment is used as an example for insufficient experimental data that is used for parameter estimation. The additional information taken from the homopolymerization experiments (Fig. 5-6) shows the significant effect on the results of the guaranteed parameter estimation if this information is also considered in the algorithm. The results of the memetic algorithm for the first experiment are shown in Fig. 7 and Fig. 8. As the information content for determining the propagation parameter space consisting of $\mathrm{k}_{\mathrm{p} 11}, \mathrm{k}_{\mathrm{p} 22}, \mathrm{r}_{1}$ and $\mathrm{r}_{2}$ is limited to the concentration profiles that are obtained from a copolymerization experiment, it is not possible to determine the three ratios. Besides the uncertainty of the measurements, the reason is that two different effects are causing the decrease of the corresponding monomer concentration, namely the homopropagation $\left(\mathrm{k}_{\mathrm{p} 11}\right.$, $\left.\mathrm{k}_{\mathrm{p} 22}\right)$ and the crosspropagation $\left(\mathrm{k}_{\mathrm{p} 12}, \mathrm{k}_{\mathrm{p} 21}\right)$. There is no possibility to distinguish the two effects and therefore one degree of freedom can be chosen freely, when trying to determine the propagation parameters. In Fig. 8 the resulting parameter space of the termination mechanism is shown. The termination rates, $\mathrm{k}_{\mathrm{tl}}$ and $\mathrm{k}_{\mathrm{t}}$, have no effect on the decrease of the concentration of both monomers and therefore the algorithm cannot determine a suitable set of $\mathrm{k}_{\mathrm{t} 1}$ and $\mathrm{k}_{\mathrm{t} 2}$, which is reflected by the resulting ranges of both parameters. The analysis of the resulting ranges enables the decision on suitable experiments to determine a more precise parameter set for the given reaction system. This knowledge cannot be generated when classical parameter estimation is performed. Every trajectory of a model with parameters from the feasible set of parameters might be a solution of the classical parameter estimation problem for a particular set of measurements. In our case study the missing information can be generated by performing two additional homopolymerization experiments
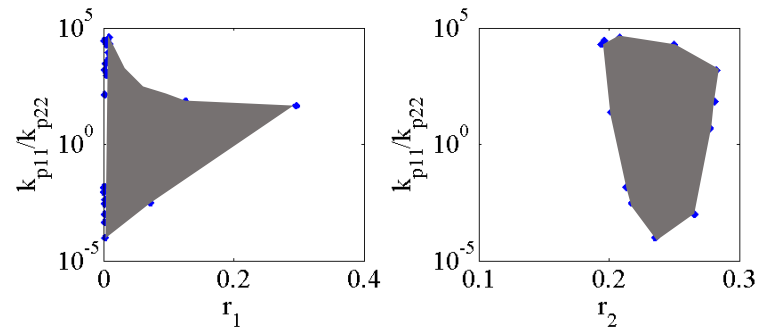

Figure 7: Set of propagation parameters calculated from the information obtained by the copolymerization experiment; true scaled values $\mathrm{r}_{1}=0$, $\mathrm{r}_{2}=0.25, \mathrm{k}_{\mathrm{p} 11} / \mathrm{k}_{\mathrm{p} 22}=0.85$

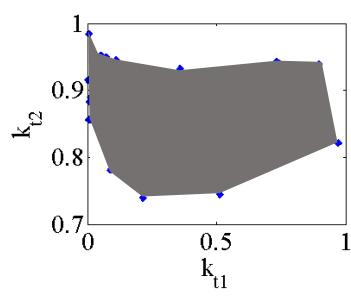

Figure 8: Set of termination parameters calculated from the information obtained by the copolymerization experiment; true scaled values: $\mathrm{k}_{\mathrm{t} 1}=0.57$, $\mathrm{k}_{\mathrm{t} 2}=0.61$

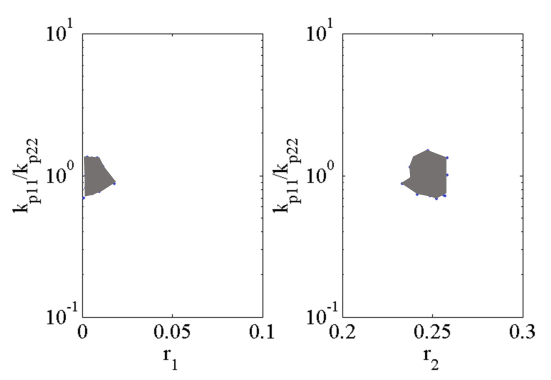

Figure 9: Set of propagation parameters calculated from the information obtained by all experiments; true scaled values $\mathrm{r}_{1}=0, \mathrm{r}_{2}=0.25, \mathrm{k}_{\mathrm{p} 11} / \mathrm{k}_{\mathrm{p} 22}=0.85$

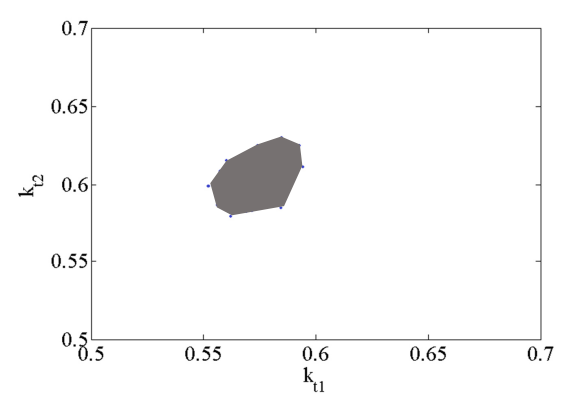

Figure 10: Set of termination parameters calculated from the information obtained by all experiments; true scaled values: $\mathrm{k}_{\mathrm{t} 1}=0.57, \mathrm{k}_{\mathrm{t} 2}=0.61$ 
and adding the information about the chain length distribution to the parameter estimation. Due to the inclusion of the concentration information of the homopolymerization experiments, the set of possible propagation parameters becomes much smaller, as these experiments provide the information about $\mathrm{k}_{\mathrm{p} 11}$ and $\mathrm{k}_{\mathrm{p} 22}$. The concentration measurements taken from the copolymerization experiment provide the information for determining both, the homo- and crosspropagation parameters. Additionally, the information about the chain length distributions enables the estimation of a smaller feasible set for the termination rates $\mathrm{k}_{\mathrm{t} 1}$ and $\mathrm{k}_{\mathrm{t} 2}$, as these parameters influence the chain length distribution but not the concentration profiles. The resulting parameter sets are shown in Fig. 9 and in Fig. 10.

The total computation time for the presented memetic algorithm, which is used to determine the 6 kinetic parameters, is 97 hours when applying a local optimization to each of the investigated 144,000 individuals. The computation time of the algorithm without applying the local optimization procedure is 6 hours. This shows that the main effort is spent for the local optimization of the individuals found during the memetic algorithm, while the time spent for the evaluation of the fitness function is negligible. The shown overall computation time of the memetic algorithm represents the time needed to achieve a fine resolution of the boundary of the feasible parameter space for the applied set of parameters (Tab. 1). The feasible areas shown in Figs. 7-10 are generated by the local optimization of all individuals. The resulting trade-off between the computation time and the resolution of the boundary can be adapted by the number of individuals to which the local optimization is applied. In Fig. 11 the difference in the resolution of the boundary of the feasible parameter set of $\mathrm{k}_{\mathrm{t} 1}$ and $\mathrm{k}_{\mathrm{t} 2}$ is shown in detail for the same situation as in Fig. 10 . The boundary is created by a linear interpolation of neighboring solutions. The number of solutions at the boundary and the values of the parameters $\mathrm{k}_{\mathrm{t} 1}$ and $\mathrm{k}_{\mathrm{t} 2}$ differ as the local optimization produces solutions on the boundary of the feasible set whereas without it more solutions lie inside the set.

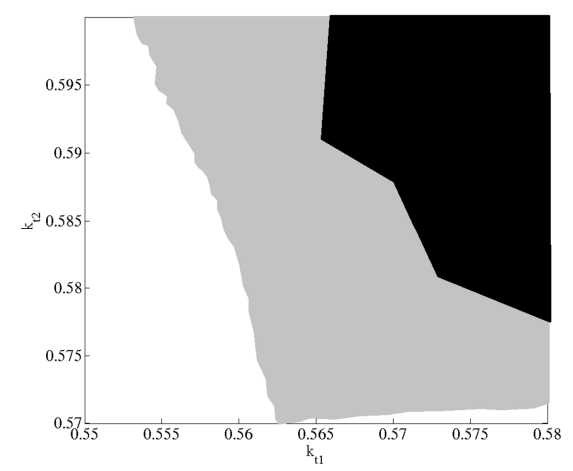

Figure 11: Resolution of the boundary of the set of scaled termination parameters calculated from the information obtained by all experiments; grey: boundary obtained from the memetic algorithm; black: boundary obtained without local optimization

\section{CONCLUSION}

The presented results show the capability of the memetic algorithm that employs a space exploring fitness evaluation to determine the feasible parameter sets for on a given set of noisy measurements in guaranteed parameter estimation. The boundaries of the feasible parameter sets result from the feasible solutions that are found. The size of the resulting parameter sets indicates whether the experimental data is sufficient or more or other experiments are needed. In the approach, the models are used as black boxes that predict the measured values, so no constraints on the type of models are present (except for the computation time needed). In further investigations, the presented algorithm will be tested with hybrid models, e.g. continuous polymerization processes with side injections, in order to show the capability to handle different types of models.

\section{ACKNOWLEDGMENT}

The research leading to these results has received funding from the European Union's Horizon 2020 research and innovation program under grant agreement $\mathrm{N}^{\circ} 636942$ „CONSENS - Integrated Control and Sensing“.

\section{REFERENCES}

[1] Walter, E. (ed.) (1990). Parameter identifications with error bound, Mathematics \& Computers in Simulation, Vol. 32. Elsevier.

[2] Paulen, R., Villanueva, M.E., Fikar, M. and Chachuat, B. (2013). "Guaranteed parameter estimation in nonlinear dynamic systems using improved bounding techniques". Proceedings of the 2013 European Control Conference (ECC'13). Zurich, Switzerland, pp. 4514-4519

[3] Kieffer, M. and Walter, E. (2011). "Guaranteed estimation of the parameters of nonlinear continuous time models: contributions of interval analysis". International Journal of Adaptive Control \& Signal Processing, vol. 25 (3), pp. 191-207

[4] Ashlock, D.;(2006); Evolutionary Computation for Modeling and Optimization; Springer Verlag, Heidelberg, Germany

[5] Urselmann, M.; Barkmann, S.; Sand, G.; Engell, S.;(2011); A Memetic algorithm for Global Optimization in Chemical Process Synthesis Problems; IEEE Transactions on Evolutionary Computation; Vol. 15, Issue 5, 659-683

[6] Goerke, T.; Kohlmann, D.; Engell; S.; (2015); Transfer of semi-batch processes to continuous processes with side injections - opportunities and limitations; under review in Macromolecular Reaction Engineering mren.201500070

[7] Rechenberg, I.; (1973) Evolutionary strategy Optmization of technical systems based on the principle of biological evolution; Evolutionsstrategie Optimierung technischer Systeme nach Prinzipien der biologischen Evolution; Frommann Holzboog, ISBN 3-7728-0373-3

[8] Etschberger, S. ; Opitz; O ; (2003); Selfadapting mutation in evolutionary strategies; Selbstadaptive Mutation bei evolutionaären Strategien; Symposium Informationswirtschaft: Ein Sektor mit Zukunft, Wien

[9] Zargar, A., Schork, F.J., (2009), Copolymer Sequence Distributions in Controlled Radical Polymerization; Macromol. React.Eng,3,118-130 WILEY-VCH Verlag GmbH \& Co. KGaA, Weinheim, Germany 